\title{
LA NECESIDAD EN EL CONCEPTO DE LA CAUSACIÓN
}

VANDA MCMURTRY

Cornell University

Central en el análisis moderno de la causación es la idea de que un efecto particular es necesitado por sus causas. Hay varias interpretaciones de esta idea, pero los filósofos que siguen la interpretación más común mantienen que una proposición causal singular implica una ley universal, es decir, que si un evento particular $e_{1}$ causa otro evento $e_{2}$, hay una ley universal de la cual $e_{1}$ y $e_{2}$ forman una instancia bajo alguna descripción de $e_{1}$ y $_{2}$. Es un error muy grave pensar que esta interpretación se acepta sin disensión, bargo es la interpretación más popular. Por consecuencia, basaré en ella esta discusión de la causación. Por ahora no me dirigiré a unos problemas con ella cuya solución no es evidente.

Anscombe y la causación sin necesidad

Una parte de la idea de que un efecto es necesitado por sus causas es la "asunción de diferencia pertinente", expresada por David Hume en su famoso dicho "misma causa, mismo efecto". G. E. M. Anscombe formula la asunción así:

If an effect occurs in one case and a similar ef. fect does not occur in an apparently similar case, there must be a relevant further difference. $^{2}$

1 Véase Jagewon Kim, "Causation, Nomic Subsumption, and the Concept of Event", Journal of Philosophy, 70, 1973, pp. 217-236.

2 G. E. M. Anscombe, "Causality and Determination", Inaugural Lecture (Cambridge: Cambridge University Press, 1971), p. 1. 
Negar que esta asunción se puede aplicar a todos los pares de efectos desemejantes es tolerar la posibilidad de la causación sin necesidad, es decir, que si un evento particular causa otro evento no es preciso que haya una ley universal de la cual estos eventos forman una instancia. Ella dice:

[A] radically different account of causation ... will deny this assumption. Such a radically opposing view can grant that often - though it is difficult to say generally when - the assumption of relevant difference is a sound principle of investigation. It may grant that there are necessitating causes, but it will refuse to identify causation as such with necessitation. It can grant that there are situations in which, given initial conditions and no interference, only one result will accord with the laws of nature; but it will not see general reason, in advance of discovery, to suppose that any given course of things has been so determined. So it may grant that in many cases difference of issue can rightly convince us of a relevant difference of circumstances; but it will deny that, quite generally, this must be so. ${ }^{3}$

Examinaré cuidadosamente las razones que Anscombe ofrece para apoyar esta concepción curiosa de la causación.

En un ensayo que pretende separar los conceptos de la causación y la necesidad es sorprendente encontrar que hay pocos ejemplos bien desarrollados de esta clase de causación. Peor todavía, las facetas esenciales del ejemplo principal no son claras. Discutamos este ejemplo de todas maneras para ver si nos debe convencer. Una bomba está conectada con un contador Geiger de tal modo que la bomba estalla si el contador Geiger llega a indicar un cierto número en su escala. Supongamos que el contadọr Geiger se coloca cerca de

3 Ibid., pp. 1-2. 
una cantidad de uranio (o que una cantidad de uranio se coloca cerca del contador Geiger) de tal manera que puede que las emisiones fortuitas de partículas subatómicas causen o no causen la indicación de ese cierto número en la escala del contador Geiger. Anscombe declara que la bomba estalla sin necesidad si estalla por cuenta de las emisiones de partículas del uranio. ${ }^{4}$ Aunque ella no la menciona, su razón por hacer tal declaración parece ser la siguiente: si la bomba estalla a causa de las emisiones de partículas del uranio, la explosión no es determinada por el colocar del contador Geiger (o del uranio), pero si la bomba estalla a causa de estas emisiones es natural decir que el colocar del contador Geiger (o del uranio) causa la explosión. Se debe notar que si de. cimos lo que es natural, tenemos que decir también que la asunción de diferencia pertinente es falsa: durante otra ocasión en la cual las condiciones iniciales se duplican exacta. mente, el colocar del contador Geiger (o del uranio) quizás no cause una explosión. Esto es posible porque las emisiones de partículas que causan la explosión son fortuitas y puede que no ocurran.

$¿$ Es verdaderamente plausible decir que un evento como el colocar del uranio es una causa de la explosión si la bomba estalla por cuenta de las emisiones de partículas del uranio? Primero queremos saber si la clase de emisiones de partículas que causan la explosión (es decir, las emisiones de una cier. ta intensidad a lo largo de una cierta trayectoria) la habrían causado de todos modos si el uranio no se hubiera colocado donde se coloca. Si esto es la verdad, seguramente el colocar del uranio es un evento superfluo al linaje causal de la explosión. Pero si esto no es la verdad, es natural pensar que el colocar del uranio es una causa de la explosión. Supóngase que el contador Geiger y la bomba se muestran al público como parte de una exposición científica (imaginemos que los directores de la exposición permiten que estas cosas se muestren para demostrar que es muy improbable que la ra.

4 Ibid., pp. 24-25. 
diación libre que se encuentra en toda región del espacio se concentre en un lugar, o algo así). Supóngase también que $A$, un científico cuyo trabajo no ganó ninguna alabanza, desea acabar con la exposición. Para realizar su deseo, $A$ coloca una cantidad de uranio al lado del contador Geiger, con eso haciendo más probable (pero no asegurando) la explosión de la bomba. Más tarde, la bomba estalla a causa de las emisiones de partículas del uranio (estas emisiones no habrían causado una explosión si el uranio no se hubiese colocado donde se colocó), y mucho daño resulta. Si $A$ es aprehendido y acusado de la destrucción de propiedad, ¿puede él mantener su inocencia diciendo que la explosión no fue causada por su acción porque no fue determinada por ella? Por supuesto, decir esto es una ridiculez. Tenemos la intuición de que la destrucción de propiedad fue una consecuencia de la acción de $A$, algo que ocasionó con su acción, y no puede evitar la responsabilidad de haberla ocasionado. Más, si $A$ no hubiera querido acabar con la exposición pero por casualidad hubiese colocado el uranio al lado del contador Geiger, no habría podido esquivar el cargo de haber actuado con negligencia. En cuanto a la responsabilidad por las consecuencias de esta acción, las autoridades deben tratar a $A$ como si hubiera causado la explosión necesitándola, por ejemplo, poniendo una piedra caliente al lado de un termóstato con el cual una bomba estaba conectada.

La característica causal de la acción de A

¿Cuál característica de la acción de $A$ es la que nos hace pensar que la acción es una causa de la explosión? Si $A$ hubiese causado la explosión necesitándola, por ejemplo, po-

5 El argumento ofrecido para establecer la inocencia de $A$ no es creíble. Consideremos un ejemplo análogo. $A$ mata a otra persona estrangulándola lentamente. Después de ser aprehendido y acusado del asesinato, $A$ dice que la muerte de su víctima no fue causada por la estrangulación porque la muerte no fue determinada por ella: cerca $\operatorname{de} A$ y su víctima había un agente libre quien libremente se decidió a no hacer nada. La acción de $A$ no determinó la muerte de la víctima, pero es una insensatez pensar que su acción no fue una causa de esa muerte. 
niendo una piedra caliente al lado de un termóstato, la respuesta sería obvia: dadas las circunstancias, tal acción habría asegurado la explosión de la bomba. Pero esta respuesta fácil no se puede dar en el ejemplo que estamos discutiendo, y luego tiene que existir otra característica cuya presencia explica nuestra intuición de que la acción de $A$ es una causa de la explosión. Quiero hallar y analizar esta característica.

Primero, se puede pensar que esta característica consiste en una cierta clase de relación de condición necesaria entre una acción del tipo que $A$ emprende y una explosión del tipo que ocurre. Naturalmente, el descubrimiento de una relación de condición necesaria aquí no significa que aislamos la característica causal (porque algunos eventos que son condiciones necesarias no son causas), ${ }^{6}$ aunque se puede pensar todavía que tal vez aislemos algo de importancia. Esta manera de ver la característica causal de la acción de $A$ parece prometer mucho, pero en realidad no cumple con su promesa. Un evento del primer tipo no es una condición necesaria de un evento del segundo tipo. Estamos imaginando que es posible que la bomba estalle en la ausencia de materia radiactiva por cuenta de la radiación libre, y aunque es muy improbable que la radiación libre se concentre en un lugar, esta concentración es un factor que no se puede eliminar completamente. Más, parece que ninguna relación de condición necesaria más débil (de una clase que aquí existe) bastará. Dada la estructura del ejemplo, una acción del tipo que $A$ emprende es seguramente una condición necesaria "práctica" de una explosión del tipo que ocurre, en el sentido de que es razonable suponer que un evento del primer tipo ocurre si se sabe que la bomba estalla pero no se sabe exactamente por qué este evento tiene lugar. La noción de una condición necesaria práctica nos atrae al principio, pero en este contexto tiene dos defectos: (1) aunque es razonable suponer que la presencia de materia radiactiva causa una explosión, puede que la radiación libre la cause de todos

6 Muchos filósofos hacen una distinción entre un evento causal y una condición causal. No hemos de preocuparnos de esta distinción aquí. 
modos; (2) puede que la presencia de materia radiactiva no sea una condición necesaria práctica de una explosión (el contador Geiger se arregla de tal manera que es muy probable que la radiación libre cause una explosión), pero que cause la explosión de todos modos. La noción de una condición necesaria práctica no nos ayuda.

Segundo, se puede pensar que esta característica consiste en una cierta clase de relación de condición suficiente entre una acción del tipo que $A$ emprende y una explosión del tipo que ocurre. Por supuesto, esta relación no puede ser de condición suficiente simpliciter: por hipótesis un evento del primer tipo no es una condición suficiente de un evento del segundo tipo. Más, parece que ninguna relación de condición suficiente más débil bastará. Supongamos que el ejemplo está construido de tal manera que una acción del tipo que $A$ emprende es una condición suficiente "práctica" de una explo. sión del tipo que ocurre, en el sentido de que es razonable suponer que la bomba estalla si se sabe que una cantidad de materia radiactiva se coloca al lado del contador Geiger pero no se sabe exactamente qué efecto tiene este evento. La idea de una condición suficiente práctica nos parece ayudar de un modo incipiente, pero en esta situación tiene dos defectos: (1) aunque es razonable suponer que la presencia de materia radiactiva cause una explosión, puede que la radiación libre la cause o que no ocurra; (2) puede que la presencia de materia radiactiva no sea una condición suficiente práctica de una explosión (el contador Geiger se arregla de tal modo que es muy improbable que la presencia de materia radiactiva cause una explosión), pero que cause la explosión de todos modos. La idea de una condición sufi. ciente práctica no nos ofrece nada.

Tercero, se puede pensar que esta característica consiste en el hecho de que una acción del tipo que $A$ emprende aumenta la probabilidad de que una explosión del tipo que ocurre tenga lugar. Por supuesto, es posible que un evento del primer tipo aumente esta probabilidad sin ser una condición necesaria práctica o suficientemente práctica de un 
evento del segundo tipo. ¿Aislamos la característica causal de la acción de $A$ aquí? Otra vez, creo que no. Supongamos que dos púgiles de habilidad igual, $B$ y $C$, se van a pelear. Dadas sus habilidades iguales, el resultado de la pelea no es cierto. Un poco antes del comienzo de la pelea los jueces imparciales que la iban a juzgar se reemplazan con jueces que favorecen a $B$. Seguramente hemos de pensar que este evento hace más probable la victoria de $B$, pero supóngase que $C$ se porta descuidadamente durante el primer período y $B$ lo vence haciéndolo caer de un solo golpe. En claro que el reemplazo injusto de los jueces no causa la victoria de $B$ aunque aumenta la probabilidad de su victoria. En consecuencia, si se sabe que los eventos de una clase aumentan la probabilidad de que los eventos de otra clase ocurran, no se puede concluir que los eventos particulares de estas dos clases se relacionan casualmente. Ahora cambiemos el ejemplo un poco. Un poco antes de la pelea $B$ oye hablar del evento que le favorece tanto, pero sabiendo que tiene la ventaja $B$ se pone tan descuidado que $C$ lo vence en el primer periodo, haciéndolo caer de un solo golpe. Es claro que el reemplazo injusto es una causa del triunfo de $C$ aunque disminuye la probabilidad de su triunfo. En consecuencia, si se sabe que los eventos particulares de dos clases de eventos se relacio. nan causalmente, no se puede concluir que los eventos de una clase aumentan la probabilidad de que los eventos de la otra clase ocurran. No vale la pena buscar la esencia de la característica causal de la acción de $A$ en la mera aumentación de la probabilidad del efecto.

Las tres sugestiones que discutimos aquí no son valiosas, pero creo que podemos aislar la característica causal que nos elude si consideramos un ejemplo análogo, es decir, el ejemplo en que la bomba está conectada con el termóstato. Causamos una explosión poniendo una piedra caliente al lado del termóstato, y es evidente por qué pensamos que el colocar de la piedra es tal causa en esta instancia: dadas las circunstancias, nuestra acción asegura que la bomba estalla. Pero si nos quedamos satisfechos con esta respuesta perde- 
mos un paralelo importante entre la eficacia causal de nues. tra acción y la de la acción de $A$. Voy a tratar de describir detalladamente este paralelo. Consideramos la "cadena" de causas inmediatas que necesitan la explosión después de nuestra acción. La explosión es causada por la indicación de un cierto número en la escala del termóstato, la cual es causada por la radiación de calor de una cierta intensidad de la piedra, la cual es causada por ciertos estados internos de la piedra. ¿Qué tiene que ver nuestra acción con esta cadena causal? Estamos suponiendo que nuestra acción no tiene nada que ver con la piedra en sí, sino que cambia la localidad de la piedra de tal manera que el calor radiado por la piedra, independientemente de la posición de ésta, se convierte en una causa de la explosión. Luego, la eficacia causal de nuestra acción se puede entender de la manera siguiente: lo que hacemos causa la existencia de una situación en que si ocurre la radiación de calor de una cierta intensidad de la piedra caliente (un evento cuya ocurrencia es causalmente independiente de nuestra acción), este evento causa la explosión de la bomba. Esta relación se puede describir también empleando el concepto de una condición: una acción del tipo que emprendemos es una condición a la vez necesaria y suficiente de la existencia de una situación en que si ocurre la radiación de calor de una cierta intensidad de la piedra, este evento causa la explosión de la bomba. Consideramos ahora la cadena de causas inmediatas que necesitan la explosión después de la acción de $A$. La explosión es causada por la indicación de un cierto número en la escala del contador Geiger, la cual es causada por las emisiones de partículas de una cierta intensidad a lo largo de una cierta trayectoria del uranio. Este último evento no es causado por nada porque es totalmente fortuito, y por consecuencia es el primer evento en la cadena de causas inmediatas que necesitan la explosión. ¿Qué tiene que ver la acción de $A$ con esta cadena causal? Otra vez estamos imaginando que la acción de $A$ no tiene nada que ver con el uranio en sí, sino que cam. bia la localidad del uranio de tal modo que las partículas 
emitidas por el uranio, independientemente de la posición de éste, se convierten en una causa de la explosión. Es interesante notar que la eficacia causal de la acción de $A$ se puede entender exactamente como se entiende la eficacia causal de nuestra acción: lo que hace $A$ causa la existencia de una situación en que si ocurren las emisiones de partículas de una cierta intensidad a lo largo de una cierta trayectoria del uranio (un evento cuya ocurrencia es causalmente independiente de todos los demás eventos previos, y a fortiori de la acción de $A$ ), este evento causa la explosión de la bomba. Esta relación se puede describir también usando el concepto de una condición: una acción del tipo que $A$ emprende es una condición a la vez necesaria y suficiente de la existencia de una situación en que si ocurren las emisiones de partículas de una cierta intensidad a lo largo de una cierta trayectoria del uranio, este evento causa la explosión de la bomba. La diferencia principal entre los dos ejemplos es la siguiente: después de nuestra acción la cadena de causas inmediatas tiene que aparecer (ya que es determinada), pero después de la acción de $A$ puede que la semejante cadena de causas inmediatas no aparezca (ya que es fortuita). En la descripción de la eficacia causal de estas acciones, ambas ocurrencias del verbo "causar" en la frase " $x$ causa la existencia de una situación en que si ocurre $y, y$ causa $z$ " tienen la connotación de necesidad. De esta observación se puede concluir que el ejemplo de Anscombe no nos muestra que tenemos un concep. to de la causación que es independiente de la necesidad o de la ley universal: la expresión de necesidad, es decir, la ley, es más compleja si la causación es de la variedad que vemos en el ejemplo de Anscombe (porque el consiguiente de la ley es condicional), pero es una ley universal de todos modos. Por otro lado, el ejemplo de Anscombe nos muestra que la relación entre los conceptos de la causación y la necesidad es más sutil de lo que uno se imagina en un principio: algunas causas necesitan sus efectos, pero otras causas los necesitan "condicionalmente". La idea de que todo efecto es necesitado por todas sus causas se debe abandonar; la idea más 
sutil, es decir, que todo efecto es necesitado o necesitado condicionalmente por cada una de sus causas se debe adoptar. Esta idea más sutil no exige que dejemos de creer que la necesidad forma una parte del concepto de la causación, y no exige que dejemos de creer que una proposición causal singular implica una ley universal.

\section{La acción de A como una causa irregular}

Si un evento causa un efecto sin necesitarlo, digamos que ese evento es una causa "irregular". En esta discusión del ejemplo de Anscombe he supuesto que nos encontramos con una instancia legítima de una causa irregular. No obstante se puede pensar que esta suposición no está bien apoyada, y como una defensa de Anscombe quiero discutir y analizar dos objeciones interesantes.

La primera objeción concede que Anscombe nos da un ejemplo de la causación, pero no concede que la causa es irregular. La objeción se puede formular fácilmente. El ejemplo de Anscombe nos convence al principio porque depende del carácter fortuito de las emisiones de partículas subatómicas del uranio. Siempre que la descripción de tal evento como fortuito se base en lo que hay en el mundo, y no sólo en la inexactitud de la medida de cantidades y distancias pequeñísimas, Anscombe tiene razón: es posible que el uranio esté en el mismo estado durante dos ocasiones, pero que emita las partículas solamente una vez. Por otro lado, si la descripción de tal evento como fortuito no se basa en lo que hay en el mundo, sino en la inexactitud de la medida de cantidades y distancias muy pequeñas, Anscombe no tiene razón: si parece que el uranio está en el mismo estado durante dos ocasiones, el hecho de que emite las partículas solamente una vez nos nuestra que hay una diferencia pertinente todavía no descubierta entre los estados que son aparentemente idénticos. Anscombe no se defiende adecuadamente de esta afirmación tenaz del determinismo universal, pero es una afirmación común. Al atacar la posibilidad de eventos for- 
tuitos, el determinista ataca también el ejemplo de Anscombe. ¿Tiene razón el determinista?

Es difícil escoger entre el determinismo y el indeterminismo. El conflicto entre estas dos doctrinas probablemente no le importa al científico si él no tiene una preferencia filosófica. Más, sea lo que sea su preferencia filosófica el científico explica o predice las emisiones de partículas subatómicas de toda materia radiactiva empleando una generalización estadística, y no una ley universal. Hace esto porque cree que es imposible descubrir tal ley (si es determinista), o que tal ley no existe ( $\mathrm{si}$ es indeterminista). El conflicto entre estas dos doctrinas puede ser un asunto de interés científico si una de las doctrinas parece ser confirmada mientras la otra parece ser desconfirmada por varios experimentos que se hacen después de progreso en la tecnología de la medi. da. Por ejemplo, supongamos que alguien inventa una máquina que da una indicación casi exacta del estado de toda materia radiactiva. Supóngase también que utilizamos esta máquina para averiguar si una cantidad de uranio, la cual emite unas partículas durante ciertas ocasiones pero no durante otras, está en el mismo estado durante todas estas ocasiones. La investigación rigurosa revela una diferencia previamente no descubierta, una diferencia que es causalmente pertinente a las emisiones de partículas. ¿Qué es lo que este descubrimiento implica? Los eventos que anteriormente se consideraban fortuitos ahora no se pueden considerar así: la aserción del indeterminista era prematura esta vez. Por otra parte, supongamos que la investigación rigurosa no revela ninguna diferencia pertinente a las emisiones de partículas. ¿Qué es lo que este descubrimiento implica? Los eventos que anteriormente se consideraban fortuitos todavía se pueden considerar así, ahora con mejor razón. La posibilidad de tal progreso en la tecnología de la medida no se puede eliminar a priori, y si este progreso ocurre puede que los resultados le favorezcan al determinista. Si pasara esto, no pensaríamos que Anscombe había descrito una instancia genuina de una causa irregular. Más, el determinista no ha 
de depender de la posibilidad de tal progreso en la tecnología de la medida para vindicar su objeción. Puede creer que el mejoramiento de la técnica de medir no es posible en realidad, y todavía puede rechazar el indeterminismo arguyendo que ya que casi todos los eventos que se experimentan de día en día parecen ser determinados por sus antecedentes, el carácter fortuito de varios eventos probablemente es una consecuencia de algún defecto irremediable en la técnica actual de medir. Este determinista está de acuerdo con la asunción de diferencia pertinente por razones inductivas, pero rechaza lo que podemos llamar la "asunción de la posibilidad de conocimiento de diferencia pertinente", es decir, la asunción de que si un efecto ocurre en una situación y un efecto semejante no ocurre en otra situación que es aparentemente idéntica, una diferencia pertinente se puede descubrir. Porque este determinista rechaza la asunción epistemológica, está de acuerdo con Anscombe cuando ella dice que la asunción de diferencia pertinente no es siempre un principio útil de investigación. No sé cómo esta especie de determinismo obstinado puede ser refutado, aunque quizás haya una refutación. Si tal refutación existe, Anscombe tiene la obligación de mencionarla para dar apoyo a su ejemplo de una causa irregular. Si tal refutación no existe, Anscombe puede mantener sólo la posibilidad lógica de la existencia de una causa irregular. Para mí esta posibilidad lógica es bastante, porque no creo que haya necesidad en el principio de que todo evento tiene una causa. El determinista a lo menos ha de mostrarnos la necesidad de este principio.

La segunda objeción es un poco más ambiciosa. Esta ob. jeción trata de establecer que Anscombe no da una instancia de una causa irregular porque no da una instancia de la causación. Esta objeción se basa en otro ejemplo que es aparentemente análogo al ejemplo de Anscombe, pero tocante al cual hay una fuerte tentación decir que no es un ejemplo de la causación. Consideremos la siguiente serie de eventos. Una persona, $D$, está sentada ante una consola en la cual hay un solo botón. $D$ cree que puede apretar el botón si desea, 
pero sin que él sepa el botón está cerrado y no se moverá si se aprieta. Para introducir la indeterminación, tenemos que suponer que $D$ tiene libre albedrío, es decir, que los resultados de las acciones intencionales de $D$ no se determinan antes de sus decisiones. Debemos suponer también que las condiciones de la acción intencional aquí son normales, es decir, que si $D$ se decide a apretar el botón éste se moverá si no está cerrado. Durante tiempo $t$ otra persona, $E$, suelta el botón para que se mueva al apretarse. Durante tiempo $t+1, D$ se decide a apretar el botón (antes de $t+i, D$ es totalmente indiferente a esta acción), y en seguida lo hace. $¿$ Es la acción de $E$ una causa del movimiento del botón? Aunque la relación de condición que obtiene entre la acción de $E$ y el movimiento del botón es idéntica a la relación de condición que obtiene entre la acción de $A$ y la explosión, es decir, una acción del tipo que $E$ emprende es una condición a la vez necesaria y suficiente de la existencia de una situación en que si $D$ se decide a apretar el botón (un evento cuya ocurrencia es causalmente independiente de la acción de $E$ ), este evento causa el movimiento del botón, no quere. mos decir que la acción de $E$ es una causa del movimiento del botón. Es importante saber por qué no queremos decir esto. Más, es necesario saber si nuestra intuición aquí amenaza la confianza que tenemos al creer que la acción de $A$ es una causa de la explosión.

¿Por qué pensamos que la acción de $E$ no es una causa del movimiento del botón? No basta replicar que pensamos esto porque hay otros eventos, por ejemplo, la decisión de $D$, que claramente lo causan: en el ejemplo de Anscombe no cabe duda de que las emisiones de partículas causan la explosión, pero este hecho no implica que la acción de $A$ no es una causa de la explosión. Quizás no pensemos que la acción de $E$ es una causa del movimiento del botón precisamente porque el primer evento en la cadena causal que determina el movimiento del botón, es decir, la decisión de $D$, es libre, y no se puede decir que un evento anterior a esta decisión causa las consecuencias de esta decisión. Si el movimiento 
del botón fuera causado por un evento que ocurrió antes de la decisión de $D$, tal vez pensásemos que el movimiento del botón no fue un resultado de una acción que $D$ libremente escogió. La sugestión que se hace aquí es la siguiente: una decisión libre es un evento anterior al cual la búsqueda de causas de los resultados de una acción intencional no puede tener éxito; una decisión libre siempre constituye el comienzo de una cadena causal. Supongamos que este es el análisis correcto de la tendencia de decir que la acción de $E$ no es una causa del movimiento del botón. ¿Cómo se puede seguir creyendo que la acción de $A$ es una causa de la explosión? Hay un paralelo interesante entre una decisión libre y un evento fortuito, a lo menos si mis definiciones son defensibles: tanto una decisión libre como un evento fortuito forman el comienzo de una cadena causal. Si esta característica de las circunstancias en las cuales $E$ actúa nos hace pensar que su acción no es una causa del movimiento del botón, quizás la misma característica de las circunstancias en las cuales $A$ actúa nos deba hacer pensar que la acción de $A$ no es una causa de la explosión. Puede que nuestras intuiciones sean distintas en los dos ejemplos porque un evento fortuito no tiene comúnmente un efecto visible (porque esta variedad de causación se experimenta sólo rara vez), y porque hay una semejanza superficial entre las acciones como la de $A$ y las acciones que verdaderamente necesitan sus efectos (como la acción de colocar una piedra caliente al lado de un termóstato con el cual una bomba está conectada).

No sé si esta segunda objeción tenga mucho impacto, y de todas maneras podemos contestarla decisivamente. Es posible que pensamos que la acción de $E$ no es una causa del movimiento del botón no porque la decisión libre de otro agente forma el comienzo de una cadena causal que necesita ese movimiento, sino porque la acción de $E$ carece de ciertas otras características. Consideremos otro ejemplo en el cual hay dos agentes y una decisión libre, pero hacia el cual nuestra actitud es totalmente diferente. Supóngase que dos personas, $F$ y $G$, se odian apasionadamente, y que $G$ desea matar a $F$. 
Para realizar su deseo, $G$ coloca una mochila llena de dinamita en la oficina de $F$ al lado de su escritorio. La mochila está arreglada de tal manera que estalla si se toca, y se coloca de tal modo que sólo $F$ la puede ver. Más tarde $F$ viene a su escritorio, se fija en la mochila, se decide a recogerla, y en seguida la toca. Por supuesto, $F$ se muere cuando la mochila estalla. ¿Podemos decir que la acción de $G$ (el colocar de la mochila letal al lado del escritorio de $F$ ) es una causa de la muerte de $F$ ? Tenemos que decir que sí, aunque la relación de condición que obtiene entre la acción de $G$ y la muerte de $F$ es idéntica a la relación de condición que obtiene entre la acción de $E$ y el movimiento del botón. Tomando en cuenta el ejemplo de $F$ y $G$, vemos por qué la segunda objeción es débil. En primer lugar, la objeción depende de un principio que es plausible prima facie, es decir, que si dos (o más) acciones libres no simultáneas desempeñan un papel posible en el linaje causal de un cierto efecto, debemos decir que la acción más propinqua al efecto en tiempo es una causa del efecto si cualquiera de las acciones es tal causa. También, la objeción depende de la suposición de que este principio se acepta por cuenta de una cierta característica de la decisión libre, es decir, que las consecuencias de una decisión libre no pueden ser causadas por un evento que ocurre anterior a la decisión. Pero vemos que este principio tiene excepciones, y la excepción ya discutida nos hace creer que no aceptamos el principio por cuenta de esa cierta característica de la decisión libre exclusivamente. Parece que aceptamos este principio de un modo limitado según las intenciones y las expectaciones de los agentes que actúan. ${ }^{7} \mathrm{La}$ segunda objeción, a lo menos como presentada aquí, no implica que Anscombe no describe una instancia legítima de una causa irregular.

7 Vemos la importancia de las intenciones y las expectaciones de los agentes si imaginamos que $F$ se da cuenta de que se morirá si toca la mochila, pero sin embargo la toca porque se decide a suicidarse. Si esto ocurriera, no pensaríamos que la acción de $G$ causa la muerte de $F$. 


\section{La causación radicalmente irregular}

Quiero dirigirme a la cuestión si tenemos un concepto de la causación que es independiente de la necesidad o la ley universal. He dicho que el ejemplo de Anscombe nos hace rechazar la idea de que todo efecto es necesitado por todas sus causas, pero no nos hace rechazar la idea de que todo efecto es necesitado o necesitado condicionalmente por cada una de sus causas. Esta última idea acomoda el ejemplo de Anscombe sin exigir la separación de la necesidad o la ley universal del concepto de la causación. Ahora supongamos que alguien sugiere que hay algunos efectos que no son necesitados por ninguna de sus causas. Tal sugestión implica el divorcio completo de la causación y la necesidad, porque una proposición singular que expresa una relación causal entre un efecto particular de esta clase y una de sus causas no im. plica ninguna ley universal. Sin duda esta sugestión es provocativa, y una indagación de sus méritos se debe emprender. Llamemos tal causación "la causación radicalmente irregular" (CRI).

En primer lugar, y como una precaución, quiero indicar un argumento que no se debe usar para establecer la posibilidad de CRI. La tesis de que una proposición causal singular implica una ley universal no es una tesis que tiene que ver con los métodos por los cuales una persona llega a saber o creer que una proposición causal singular es verdadera o falsa. Los filósofos que siguen a Hume dañan gravemente esta tesis atándola a una teoría cuestionable del reconocimiento de las series causales particulares. Muy a menudo ellos suelen ofrecer una epistemología en la cual la experiencia de la "conjunción constante" de eventos desempeña un papel necesario, pero seguramente puede que una persona reconozca una serie causal particular, en eso llegando a saber que una proposición causal singular es verdadera, sin tener ninguna experiencia de la conjunción constante de los eventos cuyas instancias observa. Esta habilidad, la de poder percibir la causación de una vez, se ejemplifica mejor cuando 
una persona es muy impresionada por la ocurrencia de un efecto y no necesita experimentar una serie semejante para convencerse que ha observado la causación. Por ejemplo, es posible que un niño no haya experimentado el desaparecimiento dramático de algodón explosivo al juntarlo con una llama (tal vez no haya experimentado nada que sea semejante a este fenómeno), pero puede que crea con justificación que el juntar del algodón con la llama causa el desaparecimiento del algodón. Si abandonamos la epistemología de conjunción constante, no vale la pena tratar de establecer la posibilidad de CRI probando que el reconocimiento de una serie causal particular no exige ninguna experiencia general. La habilidad de percibir la causación de una vez no nos muestra que tenemos un concepto de la causación que es independiente de la necesidad o la ley universal si nuestra epistemología de la causación es razonable. Por supuesto, el abandonamiento de la epistemología de conjunción constante no puede ser justificado sólo por una cierta clase de ejemplo, pero no seguiré discutiendo este asunto aquí.

Veamos si la posibilidad de CRI se puede establecer directamente empleando unos ejemplos. Tengo que confesar que nunca he oído hablar de una instancia de CRI. Es fácil comprender por qué no queremos admitir que existe CRI: si una proposición singular que expresa una relación causal no implica ninguna ley universal, ¿qué hay en la relación que nos hace pensar que la relación es causal y no accidental? Los proponentes de la posibilidad de CRI tienen una réplica lista: la causación se puede percibir de una vez. Aunque ésta es la única réplica posible (¿qué más se puede decir?), parece ser suficiente. ¿Es esta réplica en realidad suficiente? Consideremos un ejemplo putativo de CRI. ${ }^{8}$ Tenemos un cubo de vidrio de tamaño mediano, dentro del cual hay una clavija vertical $\left(\mathrm{C}_{1}\right)$. Sabemos que $\mathrm{C}_{1}$ no está pegada al cubo porque si cogemos el cubo y lo sacudimos $\mathrm{C}_{1}$ se cae. Otra clavija $\left(C_{2}\right)$ penetra la parte de arriba del cubo

8 Max Black me sugirió a mí un ejemplo semejante a éste. No estoy seguro si Black estaría de acuerdo con lo que digo tocante al ejemplo. 
de tal manera que podemos apretarla y levantarla. Sabemos también que si apretamos $\mathrm{C}_{2}$ de un modo, $\mathrm{C}_{2}$ hace contacto con $\mathrm{C}_{1}$. Queremos saber si podemos hacer que $\mathrm{C}_{1}$ se caiga pegándole con $\mathrm{C}_{2}$, así que apretamos $\mathrm{C}_{2}$. Las clavijas hacen contacto y $\mathrm{C}_{1}$ se cae. Pensamos que el apretar de $\mathrm{C}_{2}$ causó la caída de $\mathrm{C}_{1}$ porque creemos que esto fue lo que percibimos. No es difícil imaginar que esta serie de eventos es cau. sal hasta que tratemos de imaginar que es una instancia de CRI. Ahora supóngase que tratamos de hacer que $\mathrm{C}_{1}$ se caiga otra vez pegándole con $\mathrm{C}_{2}$. Apretamos $\mathrm{C}_{2}$ exactamente como la apretamos la primera vez, pero después del contacto de las clavijas $C_{1}$ no se cae. Más, supongamos que una investigación rigurosa de las dos circunstancias en las cuales apretamos $\mathrm{C}_{2}$ no revela ninguna diferencia pertinente, es decir, una diferencia que explica por qué $\mathrm{C}_{1}$ se cayó solamente una vez. Por supuesto, el segundo apretar de $C_{2}$ no causó la caída de $C_{1}$ ya que $C_{1}$ no se cayó esa vez. Es preciso saber si el primer apretar de $\mathrm{C}_{2}$ causó la caída de $\mathrm{C}_{1}$. Los antagonistas de la posibilidad de CRI dirían que sí sólo si hay una diferencia pertinente todavía no descubierta entre las dos circunstancias. Los proponentes de la posibilidad de CRI dirían que sí sin exigir que haya tal diferencia entre las dos circunstancias. Recuérdese que la base de la aserción de los proponentes consiste en la percepción de una sola serie de eventos, nada más y nada menos. Si esto no fuese la base de su aserción, ¿cómo llegaríamos a aplicar los adjetivos "causal" y "accidental" a series de eventos?

\section{Un problema con CRI: el aislamiento}

de los sistemas causales

Hagamos la asunción atrevida de que no hay una diferencia pertinente todavía no descubierta entre las dos circunstancias. ¿Es posible pensar que el primer apretar de $\mathrm{C}_{2}$ causa la caída de $\mathrm{C}_{1}$ ? Si pensamos esto, tenemos un problema interesante. Consideremos las consecuencias que este modo de pensar tiene en cuanto a la detección de la causación por 
medio de la verificación de un cierto tipo de proposición condicional. Se dice corrientemente que la causación de los eventos del tipo $e_{2}$ por los eventos del tipo $e_{1}$ se descubre si se verifica que un evento del tipo $e_{2}$ habría ocurrido durante una cierta ocasión si un evento del tipo $e_{1}$ hubiera ocurrido durante esa ocasión. El proceso que se debe seguir para hacer tal verificación se compone de un elemento pasivo (la observación) y un elemento activo (el aislamiento y la manipulación de un sistema causal). Con respecto a este elemento activo la posibilidad de CRI parece ocasionar una dificultad. Normalmente creemos que aislamos un sistema causal en el cual un evento del tipo $e_{1}$ causa otro evento del tipo $e_{2}$ únicamente si sabemos algo de los tipos de eventos que influyen en la ocurrencia de los eventos del tipo $e_{2}$, es decir, únicamente si tenemos algunas intuiciones sobre la función del sistema causal en que ocurren los eventos del tipo $e_{2}$. Un niño que no sabe nada del mecanismo que abre una puerta automáticamente llega a saber algo de los tipos de eventos que influyen el abrir de la puerta porque ve que algunos eventos (el acercarse a la puerta de una persona) son seguidos por el abrir de la puerta, mientras otros eventos (el pasar de un automóvil, el ladrar de un perro, etc.) no son seguidos por tal evento. Ya que el niño sabe que puede acercarse a la puerta si quiere, tiene la capacidad de descubrir la causación en un sistema causal que ha aislado en parte al través de la observación previa. La dificultad ocasionada por la posibilidad de CRI ahora se puede expresar de la manera siguiente: si CRI es posible, no es posible en general basar las intuiciones iniciales sobre la función de un sistema causal en la observación. En el ejemplo que acabo de desarrollar, la mera observación de que el pasar de un automóvil y el ladrar de un perro no son seguidos por el abrir de la puerta no da justificación a la creencia de que estos eventos no influyen el movimiento de la puerta cuando se abre: puede que estos eventos sean causas radicalmente irregulares del abrir de la puerta. La separación general de la necesidad o la ley universal del concepto de la causación 
hace que el aislamiento de los sistemas causales sea imposible en general. Pero si creemos que el aislamiento de los sistemas causales es imposible en general, nos vemos obligados a adoptar un escepticismo completo tocante a nuestro conocimiento de las relaciones causales en el mundo.

No creo que este primer problema con la posibilidad de CRI sea insuperable. Parece que hay varias defensas que se pueden ofrecer a favor de la posibilidad de CRI. Esta primera objeción contra la posibilidad de CRI se puede condensar del modo siguiente: (1) la observación normal de la causación presupone que aislamos los sistemas causales; (2) observamos la causación a menudo, así que aislamos los sistemas causales a menudo; (3) si CRI fuera posible, en general no seríamos capaces de aislar los sistemas causales; (4) por lo tanto, CRI no es posible. Creo que (1) no se puede dudar. La verdad de (2) es cierta también, porque un escepticismo, la que implica que casi nunca tenemos justificación si creemos que un sistema causal se ha aislado, es vacío e increíble. (3) es la premisa sospechosa. Si CRI es una posibilidad, no somos capaces de aislar los sistemas causales en general sólo si suponemos que CRI es un fenómeno común. Si resulta que CRI se manifiesta sólo en ciertas regiones del espacio o entre ciertas categorias de eventos, CRI puede existir sin imposibilitar que creamos con justificación que un sistema causal se ha aislado. Quizás sea posible creer con justificación inductiva que una causa radicalmente irregular no se mete en un sistema causal exactamente como es posible creer con justificación inductiva que una causa oculta no se mete. Puede que la primera creencia sea tan justificada como la última.

Un problema con CRI: la percepción de la causación

Ya que las ramificaciones del primer problema no parecen demasiado desagradables, fijémonos en otro problema cuyas ramificaciones son más graves y cuya solución no es tan evidente. Me parece a mí que si pensamos que el primer 
apretar de $\mathrm{C}_{2}$ causó la caída de $\mathrm{C}_{1}$ sólo porque esto fue lo que percibimos de una vez, la percepción de la causación que hicimos aquí es algo distinta de la percepción que hace el niño al ver el desaparecimiento del algodón explosivo. En verdad, lo que se está llamando "la percepción de la causación" aquí en el ejemplo de las clavijas es tan extraño que tal vez no podamos pensar que la causación es lo que se percibe. Si se puede mostrar que la causación no es lo que se percibe, la posibilidad de CRI se pondrá en peligro.

Si contemplamos el fenómeno de la sobredeterminación causal, vemos que la causación tal vez no sea lo que se percibe en el movimiento de las clavijas. Supóngase que tenemos un cubo de vidrio construído exactamente como el primer cubo. La única diferencia entre los dos cubos es la siguiente: el cubo que tenemos ahora tiene dos clavijas $\left(\mathrm{C}_{2}\right.$ y $\left.\mathrm{C}_{3}\right)$ que penetran la parte de arriba del cubo y hacen contacto con la clavija de adentro $\left(\mathrm{C}_{1}\right)$ cuando se aprietan. Varios experimentos con el cubo nuevo nos hacen pensar que el apretar de $\mathrm{C}_{2}$ es una causa radicalmente irregular de la caída de $\mathrm{C}_{1}$, mientras el apretar de $\mathrm{C}_{3}$ causa la caída de $\mathrm{C}_{1}$ con necesidad (imaginemos que si $\mathrm{C}_{1}$ se cae, se cae de la misma manera, no importa qué clavija, o combinación de clavijas, la toque). Ahora supongamos que una persona aprieta $\mathrm{C}_{2}$ mientras otra persona aprieta $\mathrm{C}_{3}$, y que cada clavija hace contacto con $\mathrm{C}_{1}$ al mismo tiempo. Por supuesto, $\mathrm{C}_{1}$ se cae. ¿Podemos decir qué fue lo que causó la caída de $\mathrm{C}_{1}$ si tomamos en cuenta sólo lo que percibimos? Si el ejemplo tuviese que ver con la causación regular, es cierto que contestaríamos afirmativamente: percibimos que tanto el apretar de $\mathrm{C}_{2}$ como el apretar de $\mathrm{C}_{3}$ causaron la caída de $\mathrm{C}_{1}$, y diríamos que la caída de $\mathrm{C}_{1}$ fue sobredeterminada. Pero, ¿podemos hacer una respuesta afirmativa si pensamos que CRI está presente? No, porque si pensamos que CRI está presente no podemos decir qué clase de relación causal percibimos. Lo que vimos en el movimiento de las clavijas puede hacernos creer que (1) el apretar de $\mathrm{C}_{3}$, pero no el de $\mathrm{C}_{2}$, causó la caída 
de $\mathrm{C}_{1}$, o (2) tanto el apretar de $\mathrm{C}_{3}$ como el de $\mathrm{C}_{2}$ causaron la caída de $\mathrm{C}_{1}$. Esta compatibilidad curiosa es una consecuencia directa de la asunción de que el apretar de $\mathrm{C}_{2}$ es una causa radicalmente irregular de la caida de $\mathrm{C}_{1}$. Este ejemplo de la sobredeterminación causal nos enseña lo siguiente: dos instancias incompatibles de la causación parecen iguales, y luego la diferencia entre ellas no se puede percibir. La sobredeterminación causal de esta variedad (es decir, en tal combinación con CRI) no es perceptible. ¿Por qué no? Una respuesta a esta pregunta nos mostrará un defecto crítico en el concepto de CRI.

Si contemplamos también el fenómeno del estorbo causal, vemos que la causación quizás no sea lo que se percibe en el movimiento de las clavijas. Supóngase que tenemos un cubo de vidrio construido exactamente con el primer cubo. La única diferencia entre los dos cubos es la siguiente: el cubo que tenemos ahora tiene un broche que estorba la caída de la clavija de adentro $\left(C_{1}\right)$. Supongamos que una persona aprieta la clavija que penetra la parte de arriba del cubo $\left(\mathrm{C}_{2}\right) . \mathrm{C}_{2}$ le pega a $\mathrm{C}_{1}$, pero por supuesto $\mathrm{C}_{1}$ no se cae. ¿Po. demos decir por qué $\mathrm{C}_{1}$ no se cayó si tomamos en cuenta sólo lo que percibimos? Si el ejemplo tuviera que ver con la causación regular, seguramente contestaríamos afirmativamente: percibimos que el broche estorbó la caída de $C_{1}$. Pero, ¿podemos hacer una respuesta afirmativa si pensamos que el apretar de $\mathrm{C}_{2}$ es una causa radicalmente irregular de la caída de $\mathrm{C}_{1}$ ? No, porque si pensamos esto no podemos decir qué fue lo que percibimos. Lo que vimos en el movimiento de las clavijas puede hacernos creer que (1) el apre$\operatorname{tar}$ de $\mathrm{C}_{2}$ no fue "eficaz" en esta instancia, y por eso el broche no estorbó (no pudo estorbar) la caída de $\mathrm{C}_{1}$ o (2) el apretar de $\mathrm{C}_{2}$ fue eficaz en esta instancia, pero el broche estorbó la caída de $\mathrm{C}_{1}$. Otra vez, una compatibilidad misteriosa es una consecuencia directa de la asunción de que el apretar de $\mathrm{C}_{2}$ es una causa radicalmente irregular de la caí. da de $C_{1}$. Este ejemplo del estorbo causal nos enseña lo siguiente: dos series incompatibles de eventos parecen iguales, 
así que la diferencia entre ellas no se puede percibir. El estorbo causal de esta categoría (es decir, en tal combinación con CRI) no es perceptible. ¿Por qué no? Otra vez, una respuesta a esta pregunta nos mostrará un defecto crítico en el concepto de CRI.

¿Por qué aparecen estas "paradojas de la percepción" si CRI se combina con la sobredeterminación y el estorbo? La respuesta es bastante clara. Consideremos el fenómeno de la sobredeterminación. Si percibimos que tanto el apretar de $\mathrm{C}_{3}$ como el apretar de $\mathrm{C}_{2}$ causan la caída de $\mathrm{C}_{1}$, percibimos que $\mathrm{C}_{1}$ se habría caído si una de las clavijas no se hubiera apretado. Consideremos el fenómeno del estorbo. Si percibimos que el broche estorba la caída de $\mathrm{C}_{1}$, percibimos que $\mathrm{C}_{1}$ se habría caído si el broche no hubiese existido. En ambos ejemplos lo que vemos justifica una aserción contraria al hecho, es decir, una aserción de lo que habría pasado si la situación actual hubiera sido diferente. Nuestra percepción es una percepción de la causación (de la sobredeterminación o el estorbo) precisamente porque justifica tal aserción contraria al hecho. Si no tenemos esta justificación (y no la tenemos en los ejemplos de la sobredeterminación y el estorbo ya discutidos), no se puede decir que percibimos la causación aunque suponemos que existe. El elemento contrario al hecho aparece más pronto en las instancias de la sobredeterminación y el estorbo que en una instancia de la causación sencilla, pero forma una parte de la causación sencilla de todas maneras. Aprieto $\mathrm{C}_{2}, \mathrm{C}_{2}$ le pega a $\mathrm{C}_{1}, \mathrm{y} \mathrm{C}_{1}$ se cae. Creo que percibo la causación aquí, pero si no percibo que $\mathrm{C}_{1}$ se habría caído si otra persona hubiera apretado $\mathrm{C}_{2}$ (y no percibo esto si creo que el apretar de $\mathrm{C}_{2}$ es una causa radicalmente irregular de la caída de $\mathrm{C}_{1}$ ), no puedo decir que percibo la causación. Lo que vi puede hacerme creer que (1) el apretar de $C_{2}$ causó la caída de $C_{1}$, o (2) el apretar de $\mathrm{C}_{2}$ no fue eficaz en esta instancia, y $\mathrm{C}_{1}$ se cayó fortuitamente. Si quitamos de la causación el elemento contrario al hecho, quitamos de la creencia de que yo percibo la causación su justificación. Sugiriendo el divorcio com. 
pleto de la causación y la necesidad, los proponedores de la posibilidad de CRI desean quitar de la causación el elemento contrario al hecho. Si tengo razón, las paradojas de la per. cepción nos prueban que tal sugestión es desastrosa. 
I begin this paper by setting forth very briefly a common thesis about causation, i.e., that causes necessitate their effects. I then contrast this view of causation as necessitation with an opposing view of causation recently offered by G. E. M. Anscombe in "Causality and Determination". The remainder of the paper is divided into five parts, and consists of an extended discussion of Anscombe's claim that there is a concept of causation in which the notion of necessitation plays no role.

First, I examine the principal example of "non-necessitating" causation described by Anscombe in order to see exactly what it is in the alleged causing event that makes one inclined to regard it as a cause. Three attempts to explain this inclination are found unsatisfactory and are dismissed. A fourth attempt is judged successful, but $\mathrm{I}$ argue that this final way of explaining why one is inclined to regard the alleged causing event as a cause does not imply that there is a concept of causation in which the notion of necessitation plays no role.

Second, I consider two possible objections to Anscombe's des. cription of her principal example. The first objection is based on an appeal to determinism, while the second is based on an analogy with free action. Against both of these potential objections I offer counterarguments on Anscombe's behalf. I conclude that her description is adequate.

Third, I attempt to construct an example in which the events are causally related only if there is a concept of causation in which the notion of necessitation plays no role. I suggest why the belief that these events are causally related has some initial plausibility.

In the final two parts I entertain two arguments which aim to show that the concept of non-necessitating causation required by my example is incoherent. The first argument is based on the apparent impossibility of isolating "causal systems," while the second is based on the apparent impossibility of perceiving causation. I maintain that the first argument is unsuccessful. I contend, however, that the second argument is conclusive. I claim that it shows very clearly the extreme difficulty inherent in the separation of the ideas of causation and necessitation. 